\title{
BMJ Open Multimorbidity in children and youth: a scoping review protocol
}

\author{
Isabella Romano, M Claire Buchan, Mark A Ferro
}

To cite: Romano I, Buchan MC, Ferro MA. Multimorbidity in children and youth: a scoping review protocol. BMJ Open 2018;8:e022413. doi:10.1136/ bmjopen-2018-022413

- Prepublication history and additional material for this paper are available online. To view these files, please visit the journal online (http://dx.doi. org/10.1136/bmjopen-2018022413).

Received 15 February 2018 Revised 23 April 2018 Accepted 3 May 2018
Check for updates

School of Public Health and Health Systems, University of Waterloo, Waterloo, Ontario, Canada

Correspondence to Isabella Romano; iromano@uwaterloo.ca

\section{ABSTRACT}

Introduction Multimorbidity (co-occurring physical and mental illness) is an important issue for clinicians and researchers with combined efforts aimed at promoting the health and well-being of individuals across the life course. In children and youth, experience of any chronic physical illness leads to a substantial increase in risk for mental illness. As a growing field of interest, research is needed to map the current state of the literature in child and youth multimorbidity in order to identify existing gaps and inform the direction of future investigations.

Methods and analysis We are proposing the conduct of a scoping review to explore the depth and breadth of existing evidence in the field of child and youth multimorbidity. The scoping review will follow the methodological framework developed by Arksey and 0'Malley, and will incorporate additional scoping review recommendations made by Levac et al. A systematic search of the following four key databases will be conducted: (1) PubMed; (2) EMBASE; (3) PsycINF0; and (4) Scopus, using combinations of Medical Subject Headings (MeSH) and Emtree terms. We will also consult grey literature sources and hand-search reference lists of included studies to identify additional studies of relevance. For eligible studies that meet all identified inclusion and exclusion criteria, a data extraction tool will be used to collect and store key study characteristics that will be relevant for collating, summarising and reporting the results of the scoping review. This scoping review also presents a novel use of quality index scoring, which we anticipate will contribute to strengthening the rigour of the scoping review methodology.

Ethics and dissemination The proposed scoping review does not require ethical approval. Final study results will be disseminated via conference presentations, publication in a peer-reviewed journal and knowledge translation activities with relevant stakeholders.

\section{INTRODUCTION}

\section{Background}

Considerable progress has been made in the domain of mental health research over the last few decades, and a sizeable body of evidence now exists surrounding the mental health of children and youth. While further research is required to decipher the complexity of mental illness among children and youth, previous work points towards several identifiable psychosocial and socioeconomic characteristics that are correlated with increased risk of mental illness in this population. A
Strengths and limitations of this study

- This proposed synthesis will be the first scoping review conducted to map the research in the field of multimorbidity (co-occurrence of physical and mental illness) among children and youth.

- The systematic search strategy will be applied to four key electronic databases using Medical Subject Headings (MeSH) and Emtree terms, and will be adapted for use in grey literature sources.

- While not a requirement of scoping reviews, this study will evaluate the methodological rigour of included studies.

- This proposed scoping review presents a feasible and efficient plan to identify gaps in, and further guide, research in child and youth multimorbidity.

socioecological framework postulated by Patalay and Fitzsimons identifies several evidence-based determinants of mental illness among children and youth, including: (1) sex and gender ${ }^{1-5}$; (2) experiences of social adversity and life stress ${ }^{1-4}$; (3) socioeconomic status ${ }^{1-5}$; (4) home environment and family relations ${ }^{2-5}$; (5) family history of mental illness ${ }^{15}$; and (6) pre-existing physical health conditions. ${ }^{1-356}$ The lattermost, physical health, is often overlooked as a key determinant of mental illness in children and youth $^{37}$; thus, it is particularly relevant to this proposed study.

Nearly $25 \%$ children are estimated to suffer from a physical illness. ${ }^{8}$ These include chronic conditions with an identifiable biological basis, and which are characterised by functional limitations as well as attempts to overcome these limitations. ${ }^{9}$ Whereas there is a variety of barriers preventing children with mental illness from accessing specialised care services, ${ }^{10}$ it has been conversely demonstrated that children and youth with physical illnesses access health services more frequently than their healthy counterparts. ${ }^{911-13}$ Like mental illness, ${ }^{14-16}$ chronic physical illnesses experienced early in life often continue into adulthood. ${ }^{17}$

It has been shown that the presence of a physical illness is a notable correlate of 
mental illness among children and youth. Interestingly, there seems to be a common underlying risk for mental illness among children with physical illness regardless of their specific diagnosis ${ }^{6}{ }^{18}$; however, little is known about the causal mechanisms or physiological factors underlying this phenomenon. ${ }^{319-21}$ Researchers have estimated that nearly half of all children and youth with a diagnosed physical illness also experience mental health problems, to an extent where between $16 \%$ and $30 \%$ are estimated to meet criteria for at least one Diagnostic and Statistical Manual-aligned diagnosis. ${ }^{3619-21}$ Typically used in gerontology to refer to the comorbidity of two or more chronic health conditions, we adopt the term, multimorbidity, to specifically describe the co-occurrence of at least one physical and at least one mental illness in children and youth. It is estimated that multimorbidity is experienced by roughly $15 \%$ of children and youth. ${ }^{3} 19-21$

\section{Rationale}

Multimorbidity should be an important area of focus for clinicians, researchers and policymaking groups, due to the implications it poses for child and youth well-being. Literature examining multimorbidity (as defined in this protocol) among adults is extensive; however, there is a paucity of studies in child and youth populations, ${ }^{3}$ much of which is unlikely to be classified as research on multimorbidity per se. To further advance the field of multimorbidity in children and youth, information is needed to identify the gaps in existing literature. This information may provide a basis for directing the focus of future multimorbidity research. To our knowledge, there is no published synthesis of the literature surrounding child and youth multimorbidity using scoping review methodology.

\section{Objectives}

The principal objective of this scoping review is to provide an overview of the existing literature on child and youth multimorbidity. Specifically, our goals are to: (1) conduct a systematic search of peer-reviewed and grey literature to scope the field of child and youth multimorbidity; (2) assess the methodological quality of studies included in the scoping review; (3) map the study characteristics, themes and methodologies used in existing child and youth multimorbidity literature; and (4) identify limitations and evidence gaps within child and youth multimorbidity literature. Information gathered from these objectives will allow us to propose recommendations to guide future research in child and youth multimorbidity.

\section{METHODS AND ANALYSIS}

The scoping review methodology has become an increasingly popular complementary epidemiological tool used in health research. ${ }^{22}$ Our scoping review will follow a six-stage framework commensurate with the following scoping review stages outlined by Arksey and O'Malley: (1) identifying the research questions; (2) identifying relevant studies; (3) selecting studies; (4) charting the data; and (5) collating, summarising and reporting the results. ${ }^{22}$ Each stage is discussed in detail below. We will also incorporate the scoping review enhancements offered by Levac et al, which further clarify and strengthen Arksey and O'Malley's framework. ${ }^{23}$ Additionally, a quality assessment exercise will be included as a component of stage 4. This scoping review protocol follows the Preferred Reporting Items for Systematic Review and Meta-Analysis Protocols checklist. ${ }^{24}$

\section{Stage 1: identifying the research questions}

We have identified one overarching research question to guide our systematic search strategy and reporting of results: 'What is known about multimorbidity in children and youth?'. This question should allow us to adequately capture the full breadth of existing literature, while providing opportunity for guiding research questions to be added or modified as required throughout the course of the study. We anticipate that an iterative process such as this will be useful as we become increasingly familiar with the literature.

We have identified several secondary questions to guide the subsequent stages of the scoping review, and to complement the broader research question above. These questions will also be subjected to iterative thinking, and will include, but will not be limited to: (1) What types of research on multimorbidity in children and youth have been conducted? (2) In which settings have previous youth multimorbidity studies been conducted? (3) Which target populations have been addressed by previous multimorbidity studies in youth? (4) What comorbid chronic physical and mental illnesses have been investigated? and (5) What identifiable questions have yet to be addressed through child and youth multimorbidity research?

\section{Stage 2: identifying relevant studies}

The second stage of the proposed scoping review will include identifying relevant studies for selection and data extraction. To do so, we will employ a systematic search strategy. The following electronic databases have been selected: (1) PubMed; (2) EMBASE; (3) PsycINFO; and (4) Scopus. In consultation with a subject-specialty liaison librarian, we have developed a PubMed-specific search strategy, reported in online supplementary file A, which generates 3226 results. The search strategy was developed to identify studies referring to the most common mental illnesses among children and youth, including anxiety, mood, conduct and attention deficit disorders. ${ }^{25} 26$ This search will be translated and tailored for use in the EMBASE, PsycINFO and Scopus databases. No date limits will be applied to the search results, but the database search query will be set to produce articles in English and within our target age group. Search results will be downloaded and imported into a citation management system, and duplicates will be immediately deleted before studies are screened for inclusion. Additionally, we will handsearch the reference lists of all literature deemed relevant 
according to the specific inclusion and exclusion criteria further outlined in stage 3 .

Following recommendations from Godin et $a l,{ }^{27}$ we will also conduct a thorough scan of relevant grey literature. First, we will scan websites of relevant organisations and agencies for any documents related to multimorbidity in children and youth. Organisations will be identified through preliminary searches online using Google, and from recommendations made by the research team and its network. Examples of such organisations may include the Offord Centre for Child Studies and the Mental Health Commission of Canada. Next, we will use existing custom Google search engines to locate documents from government sources. Two existing search engines are relevant: (1) the custom search engine for Canadian Public Health Information, created by the Ontario Public Health Libraries Association ${ }^{28}$; and (2) a custom search engine for Canadian and international government publications, created by the Maps, Data and Government Information Centre at Carleton University. ${ }^{29}$ The number of search results will be recorded at each stage of the study identification process, for both peer-reviewed and grey literature sources.

\section{Stage 3: selecting studies}

The review process will consist of two levels of screening for inclusion and exclusion criteria. Each screening level will be triangulated through use of at least two data reviewers, who will independently screen all relevant studies identified through the stage 2 systematic search of peer-reviewed and grey literature sources. The first screening process will be carried out at the level of the study title and abstract. The number of included and excluded studies at this level will be recorded, and the following decisions will be made based on screening of the title and abstract: (1) for any study that both reviewers agree to include, the study will move onto the second level of the screening process and will be read in full by each reviewer; (2) for any study that both reviewers agree to exclude, the study will not be read in full and will be excluded from the study; and (3) for any study where the decision to include or exclude is non-unanimous, or where initial screening of the study title and abstract is inconclusive, the study will also move onto the second screening level to be read in full before a final decision is made.

The second level of the study review process will require all data reviewers to obtain (ie, through library access at our institution) and read any specified articles in full to determine whether they meet the inclusion and exclusion criteria. When the decision to include or exclude an independently reviewed study is non-unanimous at the second full-screen phase, both reviewers will assess the entire study together and will discuss discrepancies to achieve a consensus. A third reviewer may be consulted for arbitration of any discordant decisions at this phase. At both the first and second phases of the review process, the number of studies included or excluded will be recorded by both reviewers. Cohen's kappa coefficient will then be calculated as a statistical measure of inter-rater reliability, and to assess the internal validity of our study's selection strategy. ${ }^{30}$

The following inclusion criteria must be met: (1) studies must refer to the multimorbidity of physical and mental illness in any clinical or non-clinical child and/ or youth population $(<18$ years old), although we recognise that the term 'multimorbidity' may likely not be used to describe such a phenomenon; (2) studies must investigate, assess or discuss the presence of mental illness in conjunction with a physical illness diagnosis; (3) studies must be published in English; (4) studies must be peer reviewed, with the exception of any grey literature in which the peer-review process may not be applicable; and (5) studies of any type of study design can be included (ie, randomised controlled trials, cohort, case-control, quasiexperimental, cross-sectional and qualitative studies).

Possible studies will be excluded from the scoping review if they investigate multimorbidity in any way other than how it has been operationally defined herein. While these inclusion and exclusion criteria will remain strict, we will also adopt an iterative approach to defining additional ad hoc criteria as the research team sees fit, and as we become increasingly familiar with the subject matter through full review of potential studies.

\section{Stage 4: charting the data}

Once studies have been identified for final inclusion, data from these studies will be collected and charted. Key characteristics of each study will be collected using a data extraction tool developed by the research team (online supplementary file B). The data extraction tool will be linked to a database for organised storage of charted data for each eligible study.

As suggested by Daudt $e t$ al, we will organise a charting trial in which a select number of studies will be charted collectively using the data extraction tool, after which reviewers will independently apply the tool to studies which have been randomly assigned to them. ${ }^{31}$ Studies will be reviewed according to a scheduled quota timeline, and reviewers will remain in constant contact throughout data collection to discuss any issues that may arise.

Additional study characteristics of interest may emerge throughout the data collection process. The research team will plan to meet on a regular basis to discuss progress during this stage, and to consider decisions regarding the relevance and adequacy of the data collection tool. Group consultations will be recorded and adequately reported, as will any changes to the study protocol that may emerge from these consultations. There is also a possibility of missing data from some eligible studies, in which case the data extraction tool will remain incomplete.

In addition to charting study characteristics using a data extraction tool, we will include a quality index score as a measurable component of each eligible study in the scoping review. The Quality Index is an appraisal tool created by Downs and Black in the context of healthcare 
and public health interventions. ${ }^{32}$ We will use a modified version of the index, previously adapted by Ferro and Speechley, ${ }^{33}$ which excludes items that are specific to intervention studies. The modified index is appropriate for the purposes of our scoping review, since our search strategy aims to capture a range of study designs possibly used in child and youth multimorbidity research. The modified index will provide an overall quality score as well as a numeric score across 15 items, and includes 'yes/no' questions that span the following sections: (1) reporting (items 1-7); (2) external validity (items 8-10); (3) internal validity (items 11-14); and (4) study power (item 15). ${ }^{34}$ We have amended item 6 to capture whether eligible studies report CIs, as well as probability values, when applicable. The modified Quality Index is available in online supplementary file $\mathrm{C}$.

Reviewers will independently rate the quality of each study and provide contextual comments. An average score will be calculated and reported in the synthesis of the final scoping review results. Ferro and Speechley's modified index has previously been used to complement study critiquing and quality assessment in systematic reviews and meta-analyses; ${ }^{34-37}$ however, to our knowledge, it has never before been included as a measure of quality in a scoping review. While quality assessment is not a typical requirement of scoping reviews, ${ }^{22} 2331$ we believe incorporating a quality index score may further strengthen the rigour of such literature syntheses.

\section{Stage 5: collating, summarising and reporting the results}

All data collected in stage 4 of the proposed scoping review will be collated, summarised and reported in such a way that maps the breadth of existing literature within the field of multimorbidity in children and youth. Studies will be classified according to their characteristics, which will have been collected through data extraction and charting, and data will be presented as tables, charts and visual maps. This will be accomplished in an aggregate manner, rather than providing an assessment of individual study qualities, to provide an overview of the research field and where it currently stands; ultimately, in an effort to identify the gaps in knowledge that currently exist.

\section{Study timeline}

Stage 1 of the proposed scoping review has been initiated for the purposes of developing this structured protocol, which is to be used as a set of guidelines outlining the aims, objectives and step-by-step methods of this study. At this point in time, stage 2 has also been initiated although only preliminarily so. We anticipate that the third stage, where eligible studies will be selected, will commence in March 2018 and will take 3 months to complete. For the first phase of screening, at the level of titles and abstracts screening, we estimate a rate of 25 total potential studies each day per reviewer. For the full-screen phase, we will aim for a combined total of 10 studies reviewed per day. Following this, stage 4 will commence. Data will be collected from all eligible studies over the course of an additional 2 months, at which point 2 final months will be allotted to stage 5 and the reporting of study results. We plan to complete the scoping review stages outlined in this protocol in a timely fashion, and expect to be completed promptly by September 2018 .

\section{Patient and public involvement}

Patients and public were neither involved in the preparation of this protocol nor will be in the final scoping review.

\section{DISCUSSION (ETHICS AND DISSEMINATION)}

Following the predefined study timeline, once all stages of the scoping review process have been completed, dissemination of findings will occur via conference presentations and the submission of results for peer-reviewed publication. Following publication of the study results, knowledge translation activities will be implemented to present findings in accessible formats (ie, infographics, fact sheets) suited to inform clinicians, policymaking groups and other stakeholders of the current state of research in child and youth multimorbidity, in addition to its future directions. Specifically, we plan to share our findings with organisations such as Children's Mental Health Ontario, the Centre for Addiction and Mental Health, and the Evidence Exchange Network for Mental Health and Addictions.

The proposed scoping review is feasible, attainable and timely. This study does not require ethics approval, as required data will be collected through the review of published peer-reviewed literature and grey literature. Results will provide unique insights concerning the extent and scope of multimorbidity research among children and youth, and will elucidate any gaps in literature that currently exist, as a means of providing guidance for future directions of research in the field.

Acknowledgements The authors acknowledge Jackie Stapleton, MLS, Liaison Librarian at the University of Waterloo, for her guidance and consultation during the preparation of the scoping review protocol. MAF holds the Canada Research Chair in Youth Mental Health.

Contributors All authors contributed meaningfully to the preparation, drafting and editing of this scoping review protocol. MAF (guarantor) conceived the idea and guided the research team at all stages of protocol development. IR (corresponding author) conceptualised the research questions, core research plan details and data extraction tool, before preparing the initial draft of this manuscript. MCB aided in developing the systematic database search framework, in consultation with a subject-specialty Liaison Librarian at the University of Waterloo. All authors approved the final submitted manuscript, after several iterations and rounds of editing, and agree to be accountable for all aspects of this protocol.

Funding This research received no specific grant from any funding agency in the public, commercial or not-for-profit sectors.

Competing interests None declared.

Patient consent Not required.

Provenance and peer review Not commissioned; externally peer reviewed.

Open Access This is an Open Access article distributed in accordance with the Creative Commons Attribution Non Commercial (CC BY-NC 4.0) license, which permits others to distribute, remix, adapt, build upon this work non-commercially, and license their derivative works on different terms, provided the original work is 
properly cited and the use is non-commercial. See: http://creativecommons.org/ licenses/by-nc/4.0/

(C) Article author(s) (or their employer(s) unless otherwise stated in the text of the article) 2018. All rights reserved. No commercial use is permitted unless otherwise expressly granted.

\section{REFERENCES}

1. Patalay P, Fitzsimons E. Correlates of mental illness and wellbeing in children: are they the same? Results from the UK millennium cohort study. J Am Acad Child Adolesc Psychiatry 2016;55:771-83.

2. Stephens T, Dulberg C, Joubert N. Mental health of the Canadian population: a comprehensive analysis [1994-1995 data]. Chronic Diseases and Injuries in Canada 1999;20:118.

3. Ferro MA. Major depressive disorder, suicidal behaviour, bipolar disorder, and generalised anxiety disorder among emerging adults with and without chronic health conditions. Epidemiol Psychiatr Sci 2016;25:462-74.

4. Merikangas KR, He JP, Brody D, et al. Prevalence and treatment of mental disorders among US children in the 2001-2004 NHANES. Pediatrics 2010;125:75-81.

5. Offord DR, Boyle MH, Racine Y. Ontario child health study: correlates of disorder. J Am Acad Child Adolesc Psychiatry 1989;28:856-60.

6. Butler A, Van Lieshout RJ, Lipman EL, et al. Mental disorder in children with physical conditions: a pilot study. BMJ Open 2018;8:e019011.

7. Merikangas KR, Nakamura EF, Kessler RC. Epidemiology of mental disorders in children and adolescents. Dialogues Clin Neurosci 2009;11:7.

8. Compas BE, Jaser SS, Dunn MJ, et al. Coping with chronic illness in childhood and adolescence. Annu Rev Clin Psychol 2012;8:455-80.

9. van der Lee JH, Mokkink LB, Grootenhuis MA, et al. Definitions and measurement of chronic health conditions in childhood: a systematic review. JAMA 2007;297:2741-51.

10. Waddell C, McEwan K, Shepherd CA, et al. A public health strategy to improve the mental health of Canadian children. Can J Psychiatry 2005;50:226-33.

11. Callahan ST, Cooper WO. Access to health care for young adults with disabling chronic conditions. Arch Pediatr Adolesc Med 2006;160:178-82.

12. Jeffrey $A E$, Newacheck PW. Role of insurance for children with special health care needs: a synthesis of the evidence. Pediatrics 2006;118: 1027-38.

13. Newacheck PW, Strickland B, Shonkoff JP, et al. An epidemiologic profile of children with special health care needs. Pediatrics 1998;102:117-23.

14. Kessler RC, Berglund P, Demler O, et al. Lifetime prevalence and age-of-onset distributions of DSM-IV disorders in the national comorbidity survey replication. Arch Gen Psychiatry 2005;62:593-602.

15. Westerhof GJ, Keyes CL. Mental illness and mental health: the two continua model across the lifespan. J Adult Dev 2010;17:110-9.

16. Woodward LJ, Fergusson DM. Life course outcomes of young people with anxiety disorders in adolescence. J Am Acad Child Adolesc Psychiatry 2001;40:1086-93.

17. Kuh $\mathrm{D}$, Shlomo YB, eds. A life course approach to chronic disease epidemiology. UK: Oxford University Press, 2004.

18. Stein RE, Bauman LJ, Westbrook LE, et al. Framework for identifying children who have chronic conditions: the case for a new definition. $J$ Pediatr 1993;122:342-7.
19. Ferro MA, Boyle MH, Avison WR. Association between trajectories of maternal depression and subsequent psychological functioning in youth with and without chronic physical illness. Health Psychol 2015;34:820-8.

20. Ferro MA, Gorter JW, Boyle MH. Trajectories of depressive symptoms in Canadian emerging adults. Am J Public Health 2015;105:2322-7.

21. Ferro MA. Mediated moderation of the relation between maternal and adolescent depressive symptoms: role of adolescent physical health. Soc Psychiatry Psychiatr Epidemiol 2015;50:1743-51.

22. Arksey H, O'Malley L. Scoping studies: towards a methodological framework. Int J Soc Res Methodol 2005;8:19-32.

23. Levac D, Colquhoun H, O'Brien KK. Scoping studies: advancing the methodology. Implement Sci 2010;5:69.

24. Shamseer L, Moher D, Clarke M, et al. Preferred reporting items for systematic review and meta-analysis protocols (PRISMA-P) 2015: elaboration and explanation. BMJ 2015;349:g7647.

25. Kessler RC, Avenevoli S, Costello EJ, et al. Prevalence, persistence, and sociodemographic correlates of DSM-IV disorders in the national comorbidity survey replication adolescent supplement. Arch Gen Psychiatry 2012;69:372-80.

26. Polanczyk GV, Salum GA, Sugaya LS, et al. Annual research review: a meta-analysis of the worldwide prevalence of mental disorders in children and adolescents. J Child Psychol Psychiatry 2015;56:345-65.

27. Godin K, Stapleton J, Kirkpatrick SI, et al. Applying systematic review search methods to the grey literature: a case study examining guidelines for school-based breakfast programs in Canada. Syst Rev 2015;4:138.

28. Ontario Public Health Libraries Association. Custom search engine for Canadian public health information. 2015 http://www.ophla.ca/ customsearchcanada.htm (accessed 7 Feb 2018).

29. Maps, Data, and Government Information Centre. Search for government publications in primo and google custom search. 2015 http://subjectguides.uwaterloo.ca/content. php?pid=408339 (accessed 7 Feb 2018).

30. Cohen J. Weighted kappa: nominal scale agreement with provision for scaled disagreement or partial credit. Psychol Bull 1968;70:213-20.

31. Daudt HM, van Mossel C, Scott SJ. Enhancing the scoping study methodology: a large, inter-professional team's experience with arksey and o'malley's framework. BMC Med Res Methodol 2013;13:48.

32. Downs SH, Black N. The feasibility of creating a checklist for the assessment of the methodological quality both of randomised and non-randomised studies of health care interventions. J Epidemiol Community Health 1998;52:377-84.

33. Ferro MA, Speechley KN. Depressive symptoms among mothers of children with epilepsy: a review of prevalence, associated factors, and impact on children. Epilepsia 2009;50:2344-54.

34. Irwin LG, Fortune DG. Risk factors for psychosis secondary to temporal lobe epilepsy: a systematic review. J Neuropsychiatry Clin Neurosci 2014;26:5-23.

35. Widjaja E, Go C, McCoy B, et al. Neurodevelopmental outcome of infantile spasms: a systematic review and meta-analysis. Epilepsy Res 2015;109:155-62.

36. Papaleontiou M, Henderson CR, Turner BJ, et al. Outcomes associated with opioid use in the treatment of chronic noncancer pain in older adults: a systematic review and meta-analysis. J Am Geriatr Soc 2010;58:1353-69.

37. Kljakovic M, Hunt C. A meta-analysis of predictors of bullying and victimisation in adolescence. J Adolesc 2016;49:134-45. 